\title{
Detecting Weather Radar Clutter by Information Fusion With Satellite Images and Numerical Weather Prediction Model Output
}

\author{
Bøvith, Thomas; Nielsen, Allan Aasbjerg; Hansen, Lars Kai; Gill, Rashpal S.; Overgaard, Søren
}

\section{Published in:}

Proceedings of the IEEE Geoscience and Remote Sensing Symposium (IGARSS) 2006

Link to article, DOI:

10.1109/IGARSS.2006.136

Publication date:

2006

Document Version

Publisher's PDF, also known as Version of record

Link back to DTU Orbit

Citation (APA):

Bøvith, T., Nielsen, A. A., Hansen, L. K., Gill, R. S., \& Overgaard, S. (2006). Detecting Weather Radar Clutter by Information Fusion With Satellite Images and Numerical Weather Prediction Model Output. In Proceedings of the IEEE Geoscience and Remote Sensing Symposium (IGARSS) 2006 IEEE. https://doi.org/10.1109/IGARSS.2006.136

\section{General rights}

Copyright and moral rights for the publications made accessible in the public portal are retained by the authors and/or other copyright owners and it is a condition of accessing publications that users recognise and abide by the legal requirements associated with these rights.

- Users may download and print one copy of any publication from the public portal for the purpose of private study or research.

- You may not further distribute the material or use it for any profit-making activity or commercial gain

- You may freely distribute the URL identifying the publication in the public portal 


\title{
Detecting Weather Radar Clutter by Information Fusion With Satellite Images and Numerical Weather Prediction Model Output
}

\author{
Thomas Bøvith*†, Allan Aasbjerg Nielsen*, Lars Kai Hansen* \\ *Informatics and Mathematical Modelling \\ Technical University of Denmark \\ DK-2800 Lyngby, Denmark \\ Email: thb@imm.dtu.dk
}

\author{
Søren Overgaard ${ }^{\dagger}$ and Rashpal S. Gill ${ }^{\dagger}$ \\ $\dagger^{\dagger}$ Danish Meteorological Institute \\ Lyngbyvej 100 \\ DK-2100 Copenhagen $\varnothing$, Denmark
}

\begin{abstract}
A method for detecting clutter in weather radar images by information fusion is presented. Radar data, satellite images, and output from a numerical weather prediction model are combined and the radar echoes are classified using supervised classification. The presented method uses indirect information on precipitation in the atmosphere from Meteosat-8 multispectral images and near-surface temperature estimates from the DMIHIRLAM-S05 numerical weather prediction model. Alternatively, an operational nowcasting product called 'Precipitating Clouds' based on Meteosat-8 input is used. A scale-space ensemble method is used for classification and the clutter detection method is illustrated on a case of severe sea clutter contaminated radar data. Detection accuracies above $90 \%$ are achieved and using an ensemble classification method the error rate is reduced by $40 \%$.
\end{abstract}

\section{INTRODUCTION}

Weather radar clutter, i.e. radar echoes from nonprecipitation targets such as man-made objects (houses, wind mills, and bridges) or the natural environment (the land and sea surface, or birds and insects in the atmosphere) are common phenomena in data from weather radars and contribute to a significantly lowered data quality. Radar clutter remains a challenge for the weather radar community, especially limiting to the application and assimilation of weather radar data into numerical weather prediction models, hydrological and other automated models [1].

Detection and mitigation of clutter have been approached with various methods from traditional signal processing techniques, e.g. Doppler velocity clutter filters (removing clutter from stationary targets) and statistical analysis of the radar data itself [2], over combining radar data with external information through data fusion [3] [4], to using new advances in radar technology, e.g. dual-polarimetric radars [5] to yield improved possibilities of distinguishing between precipitation and clutter [6].

In this contribution a method for detecting clutter in weather radar images by information fusion with new satellite based data is presented. Weather radar data are combined with multispectral satellite images from the geostationary Meteosat- 8 and model output from a numerical weather prediction (NWP) model to detect and remove weather radar clutter.

Previous studies using fusion of weather radar data and first generation Meteosat images have shown promising results [3] [7], and the improved spatio-temporal resolution of the Meteosat Second Generation (MSG) (Meteosat-8 onwards), coupled with its increased number of spectral bands, is expected to make even better detection of clutter possible.

The method is based on supervised classification of a scalespace representation of the input features and an ensemble of several classifiers is combined through a majority voting

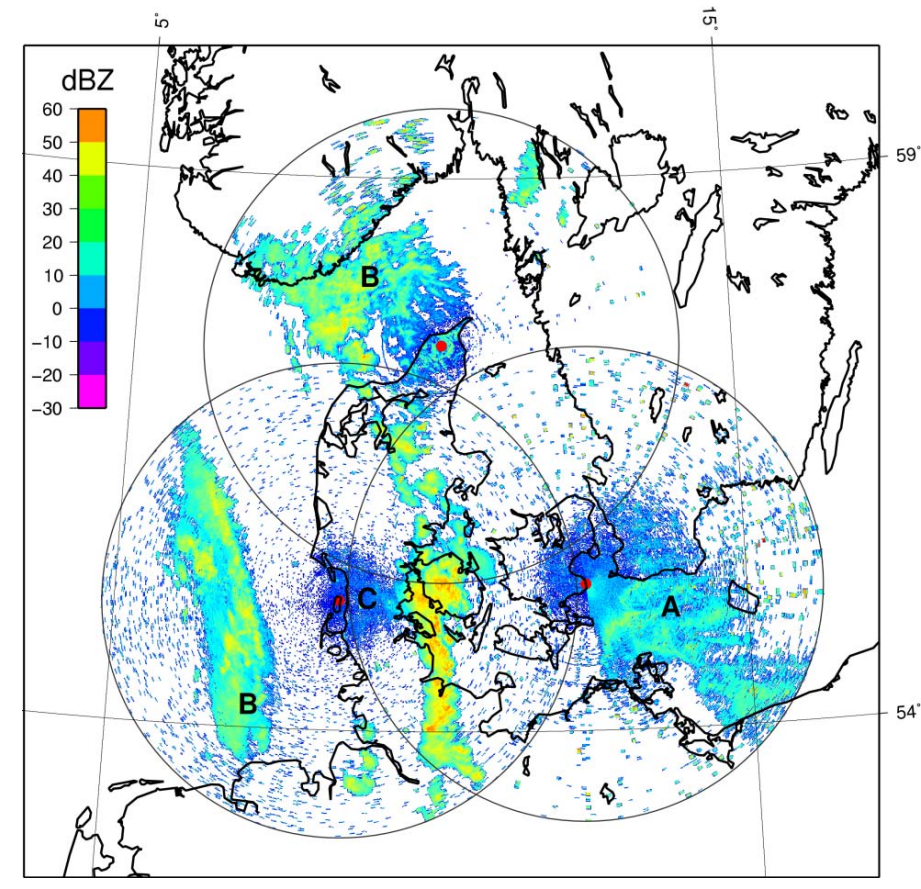

Fig. 1. Composite radar image from 2005-09-25 at 20:20 UTC. Radar reflectivity factor. A: Clutter caused by anomalous propagation. B: Precipitation. C: Close range ground clutter. Radar locations indicated by red points. Maximum range of each radar is $240 \mathrm{~km}$. 
scheme to obtain an improved classification.

\section{DATA}

\section{A. Weather radar data}

The weather radar dataset consists of data from three C-band Doppler weather radars of the Danish Meteorological Institute (DMI). The radar volume data are firstly resampled from their local polar coordinate system to a common stereographic map grid with a resolution chosen to match the range resolution of the radar data $(500 \mathrm{~m})$. The azimuth resolution of the radar data is $1^{\circ}$ which at far ranges corresponds to a spatial resolution of $4 \mathrm{~km}$. Thus the weather radar data display a varying spatial resolution from less than $500 \mathrm{~m}$ at close range increasing to $4 \mathrm{~km}$ at far range.

Example case. Severe sea clutter: The radar images acquired September 25. 2005 were heavily contaminated by sea clutter in the Baltic Sea, some close range ground clutter, as well as some electromagnetic interference clutter (Figure 1). The sea clutter was caused by anomalous propagation of the radar waves. Anomalous propagation (AP) occurs more or less frequently [8] and is the term used to describe the condition when the atmospheric refractive index does not vary normally. In a standard atmosphere the radar waves travel with increasing height in the atmosphere, however AP causes the radar waves to be bent towards and intersect with the Earth's surface producing ground clutter echoes.

On the 25. September 2005, a temperature inversion over the Baltic Sea was observed through most of the day and into the evening causing ducting (trapping) of the radar waves, which resulted in very large areas of sea clutter in the Baltic Sea off the coast from the south-eastern most radar at Stevns.

\section{B. Satellite data}

The satellite based datasets used in this study are 1) multispectral satellite images from the geostationary Meteosat-8 SEVIRI sensor and 2) an operational meteorological product derived from these images in combination with surface temperatures from a numerical weather prediction model.

Meteosat-8 SEVIRI data: The SEVIRI (Spinning Enhanced Visible and Infra-Red Imager) sensor on board Meteosat8 provides high resolution images from a geostationary orbit [9] and both the spatial and temporal resolution has been significantly improved compared to Meteosat-7. The spatial resolution at nadir is $3 \mathrm{~km}$ for the 11 visible, near infrared and thermal infrared channels and $1 \mathrm{~km}$ for the panchromatic channel. At the latitudes of Northern Europe the resolution is approximately $5 \mathrm{~km}$. The temporal resolution of the data is 15 minutes. In Figure 2 the infrared channel 4 is shown.

Precipitating Clouds: Within the 'Nowcasting SAF (Satellite Application Facility)' of EUMETSAT a range of meteorological products, e.g., cloud masks and cloud type products, are developed and produced for short-term weather forecasting. Of special interest for detection of weather radar clutter is the product named 'Precipitating Clouds'. Each pixel in the Meteosat- 8 dataset is assigned a probability of precipitation for two precipitation intensity classes, light to moderate and
ज़

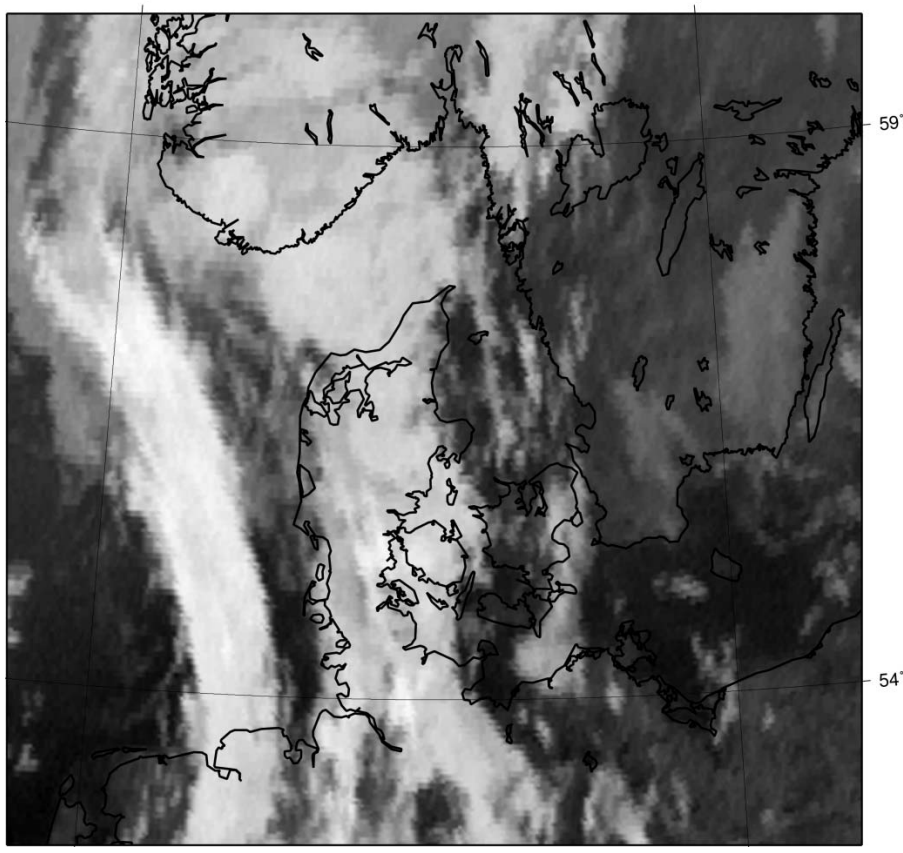

Fig. 2. Meteosat-8 SEVIRI channel 4, infrared $(3.9 \mu \mathrm{m})$. Bright shades of gray are colder (precipitating clouds), darker shades of gray are warmer.

heavy precipitation. The algorithm uses a linear combination of the spectral information of Meteosat- 8 together with surface temperatures from a NWP model, and its parameters were de-

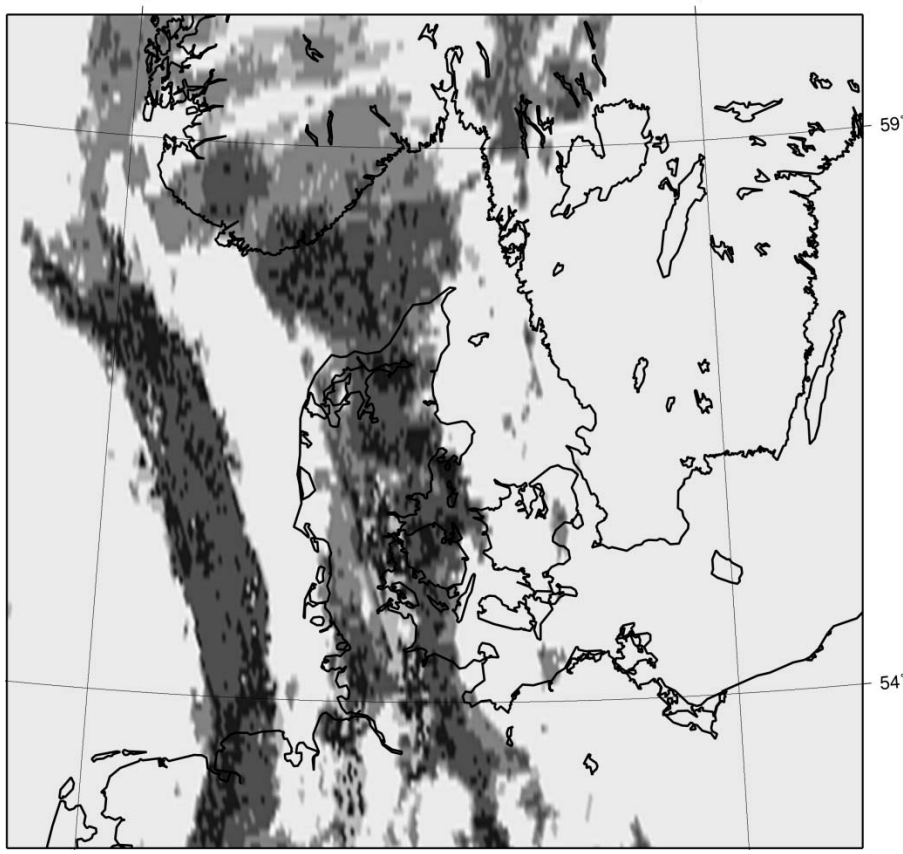

Fig. 3. 'Precipitating Clouds' product from the "Nowcasting Satellite Application Facility" of EUMETSAT. Darker colors indicate high probability of precipitation. Compare to Figure 2 showing one of the input features of the 'Precipitating Clouds' product. 
veloped and tuned using quality controlled weather radar data as described in [10]. For the classification method described in this paper the two precipitation class probabilities were added to yield the total probability of precipitation.

\section{Numerical weather prediction output}

From the numerical weather prediction model DMIHIRLAM (HIgh Resolution Limited Area Model) the near surface temperature was extracted. The highest resolution version of the DMI-HIRLAM models, the S-05 model was used. Its prediction grid consists of 41 vertical levels over a 0.05 by 0.05 degree (approximately $5 \mathrm{~km}$ ) horizontal grid.

\section{IMAGE FUSION CLASSIFICATION}

For the detection of weather radar clutter the data sources described above are combined using image fusion and then classified using manually selected training data and a scalespace ensemble classifier.

\section{A. Pixel level image fusion}

Image fusion can be performed at different levels [11] and for this study a pixel level image fusion was chosen. Fusing images on the pixel level requires resampling and geocoding of the images to a common map grid. A stereographic grid with a grid spacing of $500 \mathrm{~m}$ was chosen to match the highest resolution of the radar data. All of the datasets, the radar data, the Meteosat- 8 multispectral images, the 'Precipitating Clouds' image and the HIRLAM surface temperature fields were resampled to this grid using bicubic interpolation.

From visual inspection by superimposing the fused images, the geometrical co-registration is judged to be accurate to within the spatial resolution of the satellite image. However, since the radar and the optical multispectral sensor observe different physical phenomena (backscatter from precipitation and the cloud top, respectively), some mismatch between the estimated precipitation in the radar image and the satellite data is noticed.

Temporally the datasets are not always perfectly aligned. The radar image data are acquired every 10 minutes and the satellite data every 15 minutes. Thus a 5 minute temporal mismatch will occur for four of the six radar images per hour. The remaining two will be perfectly aligned temporally. A five minute time lag and a velocity of clouds of e.g., $10 \mathrm{~ms}^{-1}$ will result in a $3 \mathrm{~km}$ mismatch. This is below the resolution of the satellite data and thus not of great significance to the accuracy. Ideally, a temporal component should be included in the model.

\section{B. Scale-space ensemble method}

For the supervised classification, areas of precipitation and clutter in the image fused dataset is annotated and training data are extracted. The training data are divided into two groups, one for building the classifiers and one for validation of the classification results.

Before classification, an expansion of the feature space by application of a scale-space is performed. In many applications of image analysis, a scale space approach can be fruitful for image segmentation, feature extraction and classification [12]. In the development of this method it was investigated if the scale and resolution of the input image data would affect the classification result. The scale-space is made by convolution of the input features with a Gaussian kernel of a given window size corresponding to the scale.

Instead of selecting one model to classify the data, an ensemble method approach was chosen. Ensemble methods, also known as multiple classifier systems, combine several models for classification into an improved classifier with better performance than classification by use of each model individually. The data are classified using five different models: linear and quadratic discriminant analysis, a decision tree, the Mahalanobis distance and k-nearest neighbor $(\mathrm{k}=2)$ [13]. Subsequently the classification results of the five models are combined using a majority vote by which the class selected by the majority of the classifiers is chosen as the final output class.

\section{Results}

The described method was applied to two different combinations of features: The radar data in combination with the Meteosat-8 IR band number $4(3.9 \mu \mathrm{m})$ and the DMIHIRLAM-S05 surface temperature. The classification result of this feature combination is shown in Figure 4. The other combination of features was the radar data combined with the 'Precipitating Clouds' product only.

It is noticed how the large area of sea clutter in the Baltic Sea is well detected. Likewise are single pixel noise clutter and some of the ground clutter close to the radars. Errors

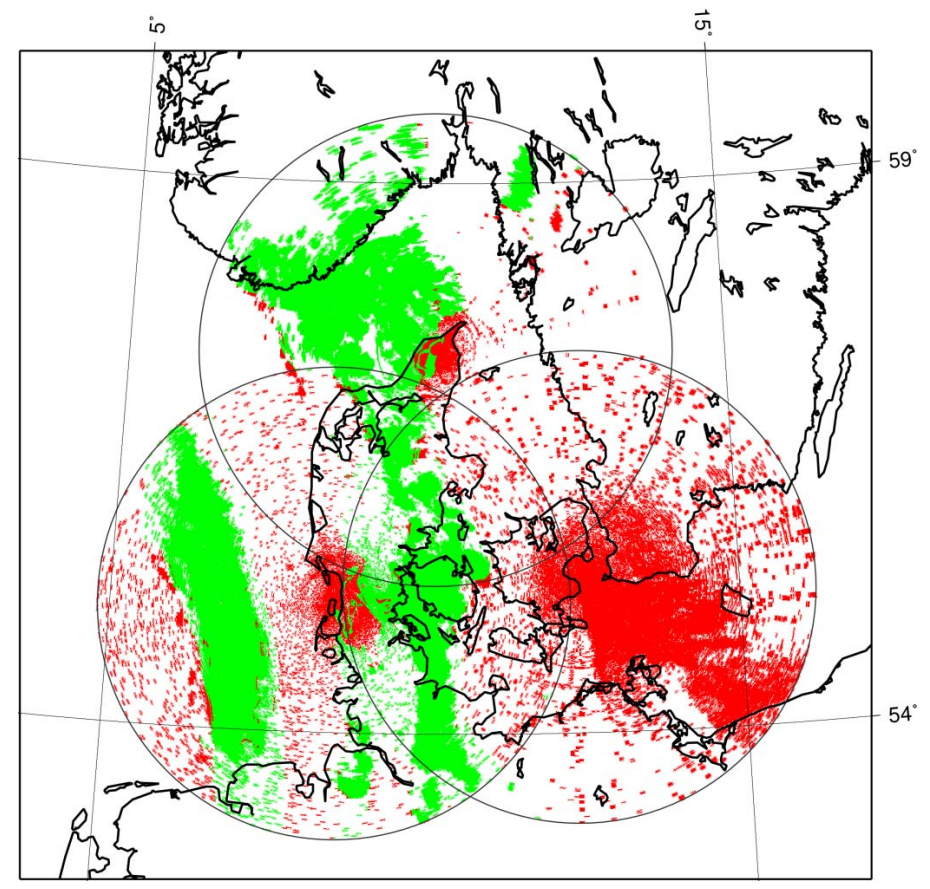

Fig. 4. Classification result. Red is clutter, green is precipitation. 


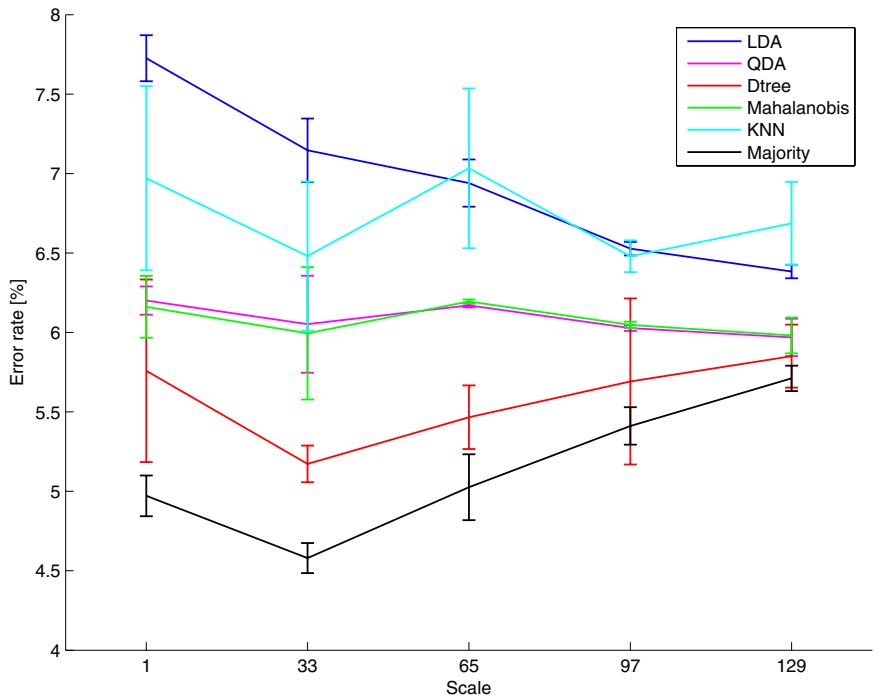

Fig. 5. Learning curves for the scale-space ensemble method. Linear discriminant, quadratic discriminant, decision tree, mahalanobis distance, $\mathrm{k}$ nearest neighbor, and majority vote. The classification was performed 3 times each with different training data and the error bars indicate the standard deviation.

occur mostly at the edges of the precipitation areas where the satellite and radar estimates do not match (precipitation is here misclassified as clutter). Other errors are found in areas of clutter close to or mixed with precipitation where clutter is misclassified as precipitation. It should be remarked that a large amount of the misclassified pixels are located in the low (close to $0 \mathrm{dBZ}$ ) backscatter ground clutter regions which can be removed by thresholding without much loss of data quality.

The classification results are assessed quantitatively by calculation of the classification error rate from the confusion matrix between the validation samples and the classified image. Error rates between $4.5 \%$ and $7.9 \%$ are seen for the example case (Figure 5). Perfect classification results, i.e., error rates close to $0 \%$ were seen for other image pairs from the same day.

From the learning curves in Figure 5 the effect of scale can be seen. The lowest classification error occurs at scale 33 (the size of the Gaussian smoothing kernel) for most of the classifiers. The reason for the increased classification accuracy at this scale is the better alignment at the edges when the input features are smoothed (i.e., seen at a coarser scale). Too much smoothing degrades the classification accuracy.

The effect of the ensemble majority vote is also seen from the learning curves: The overall error is decreased by $40 \%$ (from $7.7 \%$ to $4.6 \%$ ) by using the majority vote classifier compared to using a single linear discriminant classifier and $12 \%$ (from $5.1 \%$ to $4.5 \%$ ) compared to using a single decision tree classifier.

Classification results similar to these are seen if the 'Precipitating Clouds' product is combined with the weather radar data.

\section{CONCLUSION}

Information fusion of radar images and satellite images from Meteosat- 8 and surface temperatures from the DMIHIRLAM-S05 weather model was performed. Using supervised classification of a scale-space representation of the features and applying an ensemble classification method, weather radar clutter was detected with classification accuracies above $90 \%$. Especially, areas of widespread sea clutter caused by anomalous propagation were detected successfully. The best classification was observed at scale 33 using the majority voting scheme.

More work on the proposed method is in progress, e.g., evaluation of the performance of the method in several different meteorological situations such as convective and stratiform precipitation, as well as in situations with no precipitation. Furthermore, the development of robust model parameters based on training data from multiple radar and satellite datasets is needed before the method can be considered for operational use for removing weather radar clutter.

\section{ACKNOWLEDGMENT}

EUMETSAT is acknowledged for the operational Meteosat- 8 data and nowcasting SAF products.

\section{REFERENCES}

[1] P. Meischner, Ed., Weather Radar: Principles and Advanced Applications. Berlin, Heidelberg, Germany: Springer-Verlag, 2004.

[2] M. Steiner and J. A. Smith, "Use of three-dimensional reflectivity structure for automated detection and removal of nonprecipitating echoes in radar data," Journal of Atmospheric and Oceanic Technology, vol. 19, no. 5, pp. 673-686, 2002.

[3] D. B. Michelson and D. Sunhede, "Spurious weather radar echo identification and removal using multisource temperature information," Meteorological Applications, vol. 11, no. 1, pp. 1-14, 2004.

[4] J. A. Pamment and B. J. Conway, "Objective identification of echoes due to anomalous propagation in weather radar data," Journal of Atmospheric and Oceanic Technology, vol. 15, no. 1, pp. 98-113, 1998.

[5] V. N. Bringi and V. Chandrasekar, Polarimetric Doppler Weather Radar: Principles and Applications. New York, NY, USA: Cambridge University Press, 2001.

[6] A. V. Ryzhkov and D. S. Zrnic, "Polarimetric rainfall estimation in the presence of anomalous propagation," Journal of Atmospheric and Oceanic Technology, vol. 15, no. 6, pp. 1320-1330, 1998.

[7] G. S. Pankiewicz, C. J. Johnson, and D. L. Harrison, "Improving radar observations of precipitation with a Meteosat neural network classifier," Meteorology and Atmospheric Physics, vol. 76, no. 1-2, pp. 9-22, 2001.

[8] J. Bech, B. Codina, J. Lorente, and D. Bebbington, "Monthly and daily variations of radar anomalous propagation conditions: How "normal" is normal propagation?" in Proceedings of Second European Conference on Radar Meteorology (ERAD 2002), Delft, Netherlands, November 2002, pp. 35-39.

[9] J. Schmetz, P. Pili, S. Tjemkes, D. Just, J. Kerkmann, S. Rota, and A. Ratier, "An introduction to Meteosat second generation (MSG)," Bulletin of the American Meteorological Society, vol. 83, no. 7, pp. 977-992, 2002.

[10] "Software user manual for PGE04 of the NWCSAF/MSG: Scientific part," EUMETSAT, Satellite Application Facility to NoWCasting and Very Short Range Forecasting 1.2, 2005.

[11] C. Pohl and J. L. van Genderen, "Multisensor image fusion in remote sensing: concepts, methods and applications," International Journal of Remote Sensing, vol. 19, no. 5, pp. 823-854, 1998.

[12] T. Lindeberg, "Scale-space theory: A basic tool for analysing structures at different scales," Journal of Applied Statistics, vol. 21, no. 2, pp. 224-270, 1994.

[13] R. O. Duda, P. E. Hart, and D. G. Stork, Pattern Classification, 2nd ed. New York, NY, USA: Wiley-Interscience, 2000. 